\title{
Polarity dependence of EPR parameters for TOAC and MTSSL spin labels: Correlation with DOXYL spin labels for membrane studies
}

\author{
Derek Marsh *, Claudio Toniolo \\ Max-Planck-Institut für biophysikalische Chemie, Abt. Spektroskopie, 37077 Göttingen, Germany \\ Institute of Biomolecular Chemistry, Padova Unit, CNR, Department of Chemistry, University of Padova, 35131 Padova, Italy
}

Received 11 July 2007; revised 5 November 2007

Available online 9 November 2007

\begin{abstract}
TOAC (2,2,6,6-tetramethylpiperidine-1-oxyl-4-amino-4-carboxylic acid) is a nitroxyl amino acid that can be incorporated in the backbone of peptides. DOXYL (4,4-dimethyl-oxazolidine-1-oxyl) is a nitroxyl ring that can be attached rigidly at specific C-atom positions in the acyl chains of phospholipids. Spin-labelled phosphatidylcholines of the DOXYL type have been used previously to establish the transmembrane polarity profile in biological lipid bilayers [D. Marsh, Polarity and permeation profiles in lipid membranes, Proc. Natl. Acad. Sci. USA 87 (2001) 7777-7782]. Here, we determine the polarity dependence of the isotropic ${ }^{14} \mathrm{~N}$-hyperfine couplings, $a_{\mathrm{o}}^{\mathrm{N}}$, and $g$-values, $g_{\mathrm{o}}$, in a wide range of protic and aprotic media, for a TOAC-containing dipeptide (Fmoc-TOAC-Aib-OMe) and for a DOXYL-containing fatty acid (12-DOXYL-stearic acid). The correlation between datasets for TOAC and DOXYL nitroxides in the various solvents is used to establish the polarity profile for isotropic hyperfine couplings of TOAC in a transmembrane peptide. This calibration can be used to determine the location of TOAC at selected residue positions in a transmembrane or surface-active peptide. A similar calibration procedure is also applied to $a_{\mathrm{o}}^{\mathrm{N}}$ and $g_{\mathrm{o}}$ for the pyrroline methanethiosulphonate nitroxide (MTSSL) that is used in site-directed spin-labelling studies of membrane proteins.
\end{abstract}

(c) 2007 Elsevier Inc. All rights reserved.

Keywords: Dielectric constant; $g$-value; Hydrogen bonding; Isotropic hyperfine coupling; Nitroxide; Protic, aprotic solvents; Reaction field

\section{Introduction}

Nitroxyl spin labels are sensitive to environmental polarity and proticity via the influence of these factors on the distribution of unpaired electron spin density, $\rho_{\pi}$, in the $\mathrm{N}-\mathrm{O}$ moiety, and on the distribution and energetics of the oxygen lone-pair electrons (see e.g., [1-4]). Thus, hyperfine couplings, $a_{\mathrm{o}}^{\mathrm{N}}$, of nitroxides increase and their $g$-values, $g_{\mathrm{o}}$, decrease with increasing local dielectric constant or increasing degrees of hydrogen-bonding to the $\mathrm{N}-\mathrm{O}$ group. Griffith et al. [1] have shown for a model spin-label, di-tert-butyl nitroxide (DTBN), that the isotropic hyperfine coupling depends linearly on the reaction field

\footnotetext{
* Corresponding author. Fax: +495512011501.

E-mail address: dmarsh@gwdg.de (D. Marsh).
}

of the solvent in apolar media, but is characterised by marked non-linearity and much higher values of $a_{\mathrm{o}}^{\mathrm{N}}$ in protic media. This environmental sensitivity is an invaluable feature of spin-label EPR applications in biological systems, particularly membranes $[1,5-8]$.

Transmembrane polarity profiles have been determined at high spatial resolution in lipid bilayers by using phospholipids that were spin-labelled systematically in their hydrocarbon chains with the DOXYL nitroxide (Fig. 1) $[9,10]$. The influence of lipid composition on these transbilayer profiles has also been studied [9,11-14]. The polarity exhibits a trough-like profile across the membrane, which is registered by the hyperfine couplings and $g$-values of the DOXYL spin labels. This polarity profile not only constitutes the permeability barrier of the membrane and determines the energetics of insertion and transmembrane-anchoring of integral proteins, it also provides a 


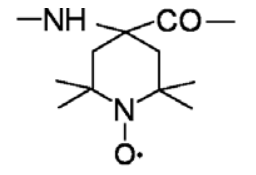

TOAC

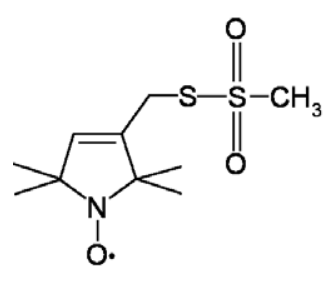

MTSSL
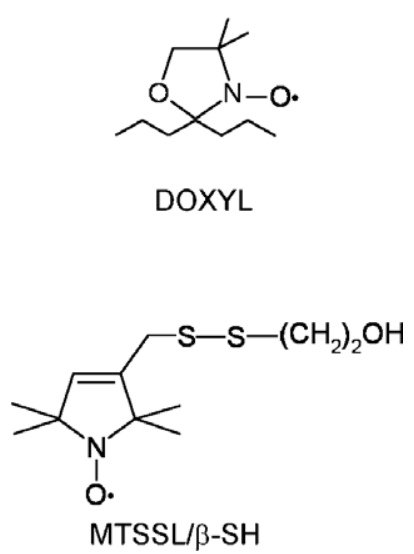

Fig. 1. Chemical structures of spin-label nitroxide moieties. TOAC (2,2,6,6-tetramethylpiperidine-1-oxy-4-amino-4-carboxylic acid) amino acid unit; DOXYL (4,4-dimethyl-oxazolidine-1-oxyl) moiety attached to an aliphatic chain; MTSSL (2,2,5,5-tetramethylpyrroline-1-oxyl-3methyl)methanethiosulphonate; MTSSL/ $\beta$-SH, $\beta$-mercaptoethanol S-S adduct of MTSSL.

means by which the location of spin-labelled peptides and proteins may be determined in membranes. Determining the vertical location within the membrane of other spinlabelled lipid species by this method is straightforward (see e.g., $[12,13,15])$. However, the DOXYL nitroxides that are used with lipids are not those normally employed in the spin-labelling of peptides or proteins for EPR studies. Consequently, it is necessary to establish correlations relating the EPR properties of DOXYLs to those of the nitroxyl reagents that are used for spin-labelling peptides and proteins.

TOAC (see Fig. 1) is a nitroxyl $\mathrm{C}^{\alpha}$-tetrasubstituted amino acid that can be used in peptide synthesis. The nitroxide ring of TOAC is incorporated rigidly in the peptide backbone and therefore is optimally situated to determine the location of the various secondary structural elements of the peptide. Sensitivity of the isotropic hyperfine couplings to membrane location has been demonstrated for TOAC-labelled trichogin GA IV [16] and alamethicin F50/5 $[17,18]$ antimicrobial peptaibols. Positional specificity in nitroxide-labelling of proteins is now obtained routinely in site-directed spin-labelling [19-21]. This is achieved by cysteine-substitution mutagenesis, accompanied by spin-labelling with the nitroxyl $\mathrm{S}-\mathrm{S}$ reagent MTSSL (see Fig. 1). Such site-directed nitroxide scanning has been applied with considerable success to study the environmental polarity of membrane-embedded helices in bacteriorhodopsin $[8,22,23]$ and in colicin A [24].

In the present work, we determine isotropic hyperfine coupling constants and $g$-values for representative TOAC and DOXYL nitroxide spin labels in a wide range of both protic and aprotic solvents. From the dependence of $a_{\mathrm{o}}^{\mathrm{N}}$ and $g_{\mathrm{o}}$ on the polarisation reaction field in aprotic solvents, and on the extent of hydrogen bonding in protic solvents, it is possible to correlate the polarity dependence of TOAC spin labels with those of DOXYL spin labels. Thus, a cal- ibration is established for the transmembrane profile of $a_{\mathrm{o}}^{\mathrm{N}}$ for TOAC, which can be used to locate the position and orientation of TOAC-containing peptides in membranes. From published results on the polarity dependence of $a_{\mathrm{o}}^{\mathrm{N}}$ and $g_{\mathrm{o}}$ for MTSSL [25], a calibration is also established for the transmembrane location of cysteine residues in site-directed spin-labelling.

\section{Theoretical background}

\subsection{Isotropic hyperfine couplings}

The isotropic ${ }^{14} \mathrm{~N}$-hyperfine coupling constant, $a_{\mathrm{o}}^{\mathrm{N}}$, of a nitroxide is related to the unpaired electron spin densities on the nitrogen $\left(\rho_{\pi}^{\mathrm{N}}\right)$ and the oxygen $\left(\rho_{\pi}^{\mathrm{O}}\right)$ by a McConnell-type relation [26]:

$a_{\mathrm{o}}^{\mathrm{N}}=Q_{\mathrm{N}} \rho_{\pi}^{\mathrm{N}}+Q_{\mathrm{NO}} \rho_{\pi}^{\mathrm{O}}$

where $\rho_{\pi}^{\mathrm{N}}+\rho_{\pi}^{\mathrm{O}} \approx 1$, and the leading term is that involving $Q_{\mathrm{N}}\left(\gg Q_{\mathrm{NO}}\right)$. The local polarisation electric field from the solvent (or an external electric field) induces redistribution of the unpaired electron spin density within the nitroxide moiety. To first order, the change in spin density on the nitrogen, $\Delta \rho_{\pi}^{\mathrm{N}}$, depends linearly on the electric field component, $E_{x}$, along the $\mathrm{N}-\mathrm{O}$ bond [1]:

$\Delta \rho_{\pi}^{\mathrm{N}}=C_{1} E_{x}$

Simple Hückel molecular orbital theory predicts the strength of the redistribution to be given by $C_{1}=1.5 \times$ $10^{-11} \mathrm{~V}^{-1} \mathrm{~m}[1]$. Ab initio calculations have produced somewhat lower values: $C_{1}=1.2-1.3 \times 10^{-11} \mathrm{~V}^{-1} \mathrm{~m}$ [27], and semi-empirical molecular orbital methods have predicted a considerably lower sensitivity: $C_{1}=0.5 \times 10^{-11} \mathrm{~V}^{-1} \mathrm{~m}$ [4]. On the other hand, changes in experimental coupling constants, on titration of a dissociable group attached to the nitroxide ring, are consistent with a value of $C_{1}=1.9 \times$ $10^{-11} \mathrm{~V}^{-1} \mathrm{~m}$, for $Q_{\mathrm{N}}-Q_{\mathrm{NO}}=2.04 \mathrm{mT}$, when compared with classical electrostatic calculations of the change in local electric field [28].

In aprotic media, the reaction field at the nitroxide that arises from polarisation of the solvent by the (polarisable) $\mathrm{N}-\mathrm{O}$ electric dipole, $p$, is given by $[29,30]$ :

$E_{R, x}=\frac{1}{4 \pi \varepsilon_{\mathrm{o}}} \frac{f\left(\varepsilon_{\mathrm{r}}\right)}{1-\left(\frac{n_{D}^{2}-1}{n_{D}^{2}+2}\right) f\left(\varepsilon_{\mathrm{r}}\right)} \cdot \frac{p}{r_{\text {eff }}^{3}}$

where $\varepsilon_{\mathrm{r}}$ is the relative dielectric permittivity of the solvent, $\varepsilon_{\mathrm{O}}$ is the permittivity of free space, $n_{\mathrm{D}}$ is the refractive index of the pure nitroxide ( $n_{\mathrm{D}}^{2} \approx 2.0$; see e.g., [31]), and $r_{\text {eff }}$ is the effective interaction molecular radius of the spin label. Onsager's model for a dipole of molecular radius $r_{\text {eff }}$ embedded in a homogeneous dielectric yields: $f\left(\varepsilon_{\mathrm{r}}\right)=$ $2\left(\varepsilon_{\mathrm{r}}-1\right) /\left(2 \varepsilon_{\mathrm{r}}+1\right)$. With an exponential transition to the bulk dielectric constant that depends inversely on radial distance, instead of a step function at the molecular surface, the Onsager result is modified to [32]: 
$f\left(\varepsilon_{\mathrm{r}}\right)=\frac{3 \varepsilon_{\mathrm{r}} \ln \varepsilon_{\mathrm{r}}}{\varepsilon_{\mathrm{r}} \ln \varepsilon_{\mathrm{r}}-\varepsilon_{\mathrm{r}}+1}-\frac{6}{\ln \varepsilon_{\mathrm{r}}}-2$

This improved reaction field, which will be adopted here, saturates less rapidly than that of Onsager, and best describes the polarity dependence of spin-label EPR parameters [30]. For $\varepsilon_{\mathrm{r}}$ close to unity Eq. (4) becomes: $f\left(\varepsilon_{\mathrm{r}}\right) \approx \frac{1}{6} \ln \varepsilon_{\mathrm{r}}$ [31], confirming that $E_{R, x}=0$ for $\varepsilon_{\mathrm{r}}=1$. Combining Eqs. (1)-(3), the isotropic ${ }^{14} \mathrm{~N}$-hyperfine coupling of a nitroxide in an aprotic solvent is then given by (cf. [1]):

$a_{\mathrm{o}}^{\mathrm{N}}=a_{\mathrm{o}}^{\varepsilon=1}+K_{v} \frac{f\left(\varepsilon_{\mathrm{r}}\right)}{1-\frac{1}{4} f\left(\varepsilon_{\mathrm{r}}\right)}$

where $a_{\mathrm{o}}^{\varepsilon=1}$ is the extrapolated isotropic coupling constant of the nitroxide in a medium of relative dielectric permittivity $\varepsilon_{\mathrm{r}}=1$. The coefficient of the polarity-dependent term is given by:

$K_{v}=\frac{1}{4 \pi \varepsilon_{\mathrm{o}}}\left(Q_{\mathrm{N}}-Q_{\mathrm{NO}}\right) C_{1} \frac{p}{r_{\mathrm{eff}}^{3}}$

which depends solely on the properties of the particular nitroxide spin-label.

For protic media, the effects of hydrogen bonding on the isotropic hyperfine couplings far outweigh those of the solvent polarity $[1,33,34]$. In the presence of a proton donor, chemical exchange takes place between free and hydrogen-bonded nitroxides, which have isotropic ${ }^{14} \mathrm{~N}$-hyperfine couplings $a_{\mathrm{o}, \mathrm{o}}^{\mathrm{N}}$ and $a_{\mathrm{o}, \mathrm{h}}^{\mathrm{N}}$, respectively. Because exchange is fast compared with the difference in hyperfine couplings, the isotropic coupling constant that is observed experimentally is given by [35]:

$a_{\mathrm{o}}^{\mathrm{N}}=\left(1-f_{\mathrm{h}}\right) a_{\mathrm{o}, \mathrm{o}}^{\mathrm{N}}+f_{\mathrm{h}} a_{\mathrm{o}, \mathrm{h}}^{\mathrm{N}}$

where $f_{\mathrm{h}}$ is the fractional population of hydrogen-bonded nitroxides. The relations given by Eqs. (5) and (7) form a basis on which the polarity dependence of one nitroxide can be referred to that of another. For membrane applications, we wish to refer other spin labels to the DOXYL nitroxide with which polarity profiles have been established [9].

\subsection{Anisotropic hyperfine couplings}

The polarity dependence of the complete hyperfine tensor is determined by that of both the isotropic and anisotropic (i.e., dipolar) terms. For the diagonal $z$-element:

$A_{z z}=a_{\mathrm{o}}^{\mathrm{N}}+2\left|T_{\perp}^{\mathrm{d}}\right|$

where $-\left|T_{\perp}^{\mathrm{d}}\right|$ is the perpendicular element of the traceless hyperfine tensor that arises from the electron-nucleus dipolar interaction. Clearly, the latter depends directly on the unpaired spin density on the nitrogen: $T_{\perp}^{\mathrm{d}}=T_{\perp . \mathrm{o}}^{\mathrm{d}} \rho_{\pi}^{\mathrm{N}}$, where $T_{\perp, \mathrm{o}}^{\mathrm{d}}$ is the value of $T_{\perp}^{\mathrm{d}}$ for $\rho_{\pi}^{\mathrm{N}}=1$. Combining Eqs. (1) and (8), $A_{z z}$ is related to the isotropic coupling constant, $a_{\mathrm{o}}^{\mathrm{N}}$, by:
$A_{z z}=\left(1+\frac{2\left|T_{\perp, \mathrm{o}}^{\mathrm{d}}\right|}{Q_{\mathrm{N}}-Q_{\mathrm{NO}}}\right) a_{\mathrm{o}}^{\mathrm{N}}-\frac{2\left|T_{\perp, \mathrm{o}}^{\mathrm{d}}\right| Q_{\mathrm{NO}}}{Q_{\mathrm{N}}-Q_{\mathrm{NO}}}$

which predicts a linear dependence. The value of $\left|T_{\perp .0}^{\mathrm{d}}\right|=$ $\left(\mu_{\mathrm{o}} / 10 \pi\right) h^{-1} g_{\mathrm{N}} \beta_{\mathrm{N}} g_{\mathrm{e}} \beta_{\mathrm{e}}\left\langle r_{2 \mathrm{p}}^{-3}\right\rangle$ is $47.8 \mathrm{MHz}(\approx 1.70 \mathrm{mT})$ for a ${ }^{14} \mathrm{~N} 2 \mathrm{p}$ orbital [36]. Values of $Q_{\mathrm{N}}$ and $Q_{\mathrm{NO}}$ depend on the particular nitroxide and may be estimated by using Eq. (9) [37].

\subsection{Isotropic g-values}

The $g$-factors of nitroxides depend on the spin density, $\rho_{\pi}^{\mathrm{O}}$, on the oxygen atom and additionally on the energies and distribution of the lone pair orbitals. Therefore, as for the hyperfine couplings, the isotropic $g$-values respond to environmental polarity and hydrogen bonding. The sign of the polarity dependence is, however, opposite to that of the hyperfine coupling.

The major contribution to the polarity dependence of the nitroxide $g$-tensor comes from the $g_{x x}$ element. This is given from Stone's perturbation treatment $[2,3]$ :

$g_{x x}=g_{\mathrm{e}}+\frac{2 \zeta_{\mathrm{O}}\left(C_{\mathrm{O}, y}^{(n)}\right)^{2} \rho_{\pi}^{\mathrm{O}}}{\Delta E_{n \pi^{*}}}$

where $g_{\mathrm{e}}=2.002319$ is the free-electron $g$-value, $\zeta_{\mathrm{O}}$ is the spin-orbit coupling of oxygen, $C_{\mathrm{O}, y}^{(n)}$ is the coefficient of the oxygen $2 \mathrm{p}_{y}$ orbital in the lone pair orbital, and $\Delta E_{n \pi *}$ is the $n \rightarrow \pi^{*}$ excitation energy. If the lone pair is confined to the $2 \mathrm{p}_{y}$ orbital on the oxygen (see [8]), $g_{x x}$ is the only $g$ tensor element that depends on the lone pair, and $g_{y y}$ then depends only on the orbitals of the $\mathrm{N}-\mathrm{O}$ bond. The lone pair orbital is affected solely by hydrogen bonding and not by environmental polarity. The $g_{y y}$ tensor element is considerably less sensitive to polarity and hydrogen bonding than is the $g_{x x}$ element because the energy denominators for $g_{y y}$ involve the bonding and antibonding $\mathrm{N}-\mathrm{O}$ $\sigma$-orbitals, which do not lie as close in energy to the unpaired electron orbital as does the lone pair. The $g_{z z}$ tensor element, in turn, is practically insensitive to polarity and hydrogen bonding $\left(g_{z z} \approx g_{\mathrm{e}}\right)$.

The isotropic $g$-value is the trace of the $g$-tensor: $g_{\mathrm{o}}=(1 / 3)\left(g_{x x}+g_{y y}+g_{z z}\right)$. Consequently, from Eq. (10), changes in $g$-value, $\delta g_{\mathrm{o}}$, are related to the polarity and hydrogen-bonding-dependent terms by (cf. [38,39]):

$\frac{\delta g_{\mathrm{o}}}{g_{\mathrm{o}}-g_{\mathrm{e}}}=\frac{\delta \rho_{\pi}^{\mathrm{O}}}{\rho_{\pi}^{\mathrm{O}}}-\frac{\delta \Delta E_{n \pi^{*}}}{\Delta E_{n \pi^{*}}}+\frac{\delta\left(C_{\mathrm{O}, y}^{(n)}\right)^{2}}{\left(C_{\mathrm{O}, y}^{(n)}\right)^{2}}+\frac{\delta g_{y y}}{g_{y y}}$

where the first two terms on the right are likely to dominate [2]. In contrast, changes in the isotropic hyperfine coupling depend only on the unpaired spin density (see Eq. (1)). Therefore $g$-values are expected to be preferentially sensitive to hydrogen-bonding, as compared with hyperfine couplings.

The $g$-factor, like the hyperfine coupling, also responds approximately linearly to the polarisation reaction field (see e.g., [4,27]). Consequently, the polarity dependence 
of the isotropic $g$-value in aprotic solvents is given similarly by (cf. Eq. (5)):

$g_{\mathrm{o}}=g_{\mathrm{o}}^{\varepsilon=1}+K_{v, g} \frac{f\left(\varepsilon_{\mathrm{r}}\right)}{1-\frac{1}{4} f\left(\varepsilon_{\mathrm{r}}\right)}$

where $g_{\mathrm{o}}^{\varepsilon=1}$ is the isotropic $g$-factor in a medium of relative dielectric permittivity $\varepsilon_{\mathrm{r}}=1$, and $K_{v, g}$ is a constant for a particular nitroxide.

As with the hyperfine couplings, in protic media the effects of hydrogen bonding on the $g$-values considerably outweigh those of the solvent polarity. Because line shifts arising from $g$-value differences are small compared with the magnitude of the overall resonance field, fast chemical exchange between free and hydrogen-bonded nitroxides effectively averages the $g$-values. An expression similar to that for the hyperfine couplings therefore also holds for the isotropic $g$-values in protic solvents (cf. Eq. (7)):

$g_{\mathrm{o}}=\left(g_{\mathrm{o}, \mathrm{h}}-g_{\mathrm{o}, \mathrm{o}}\right) f_{\mathrm{h}}+g_{\mathrm{o}, \mathrm{o}}$

where $g_{\mathrm{o}, \mathrm{h}}$ and $g_{\mathrm{o}, \mathrm{o}}$ are the isotropic $g$-values of the hydrogen-bonded and free nitroxides, respectively, and $f_{\mathrm{h}}$ is the fraction of nitroxides that are hydrogen-bonded.

\section{Results and discussion}

\subsection{Polarity dependence of isotropic hyperfine couplings}

Fig. 2 shows the dependence of the isotropic nitrogen hyperfine coupling, $a_{\mathrm{o}}^{\mathrm{N}}$, on the strength of the reaction field of the solvent, $f\left(\varepsilon_{\mathrm{r}}\right) /\left(1-\frac{1}{4} f\left(\varepsilon_{\mathrm{r}}\right)\right)$, for the TOAC and DOXYL spin labels, where $\varepsilon_{\mathrm{r}}$ is the relative dielectric permittivity of the solvent. As found previously for DTBN [1], the data for each spin label fall into two groups: those corresponding to protic solvents and those to aprotic solvents. The values of $a_{\mathrm{o}}^{\mathrm{N}}$ for aprotic solvents are lower than those for protic solvents and increase approximately linearly with the strength of the polarisation reaction field of the solvent. Solid lines in Fig. 2 are linear regressions to the data for aprotic solvents. Note that, in this context, most amines and amides are grouped with the aprotic solvents. Apparently these are unable to hydrogen-bond effectively with the nitroxide, unlike the hydroxylic solvents which are classified as protic. Values for the gradient, $K_{v}$, and intercept, $a_{\mathrm{o}}^{\varepsilon=1}$, according to Eqs. (4) and (5) are given for aprotic solvents in Table 1.

The values of $a_{\mathrm{o}}^{\mathrm{N}}$ for protic solvents are considerably higher than those for aprotic solvents and show a steep non-linearity that is attributable to hydrogen bonding. However, the values of $a_{\mathrm{o}}^{\mathrm{N}}$ for the more apolar protic solvents depend linearly on $f\left(\varepsilon_{\mathrm{r}}\right) /\left(1-\frac{1}{4} f\left(\varepsilon_{\mathrm{r}}\right)\right)$ with a gradient similar to that for aprotic solvents (dashed lines in Fig. 2). The parameters of the linear regressions for protic solvents (dashed lines) are also included in Table 1.

Isotropic hyperfine coupling constants for the MTSSL spin-label (both alone and coupled to $\beta$-mercaptoethanol, MTSSL/ $\beta-\mathrm{SH}$ ) have been recorded in solvents of different polarities by Owenius et al. [25]. These data show a depen-

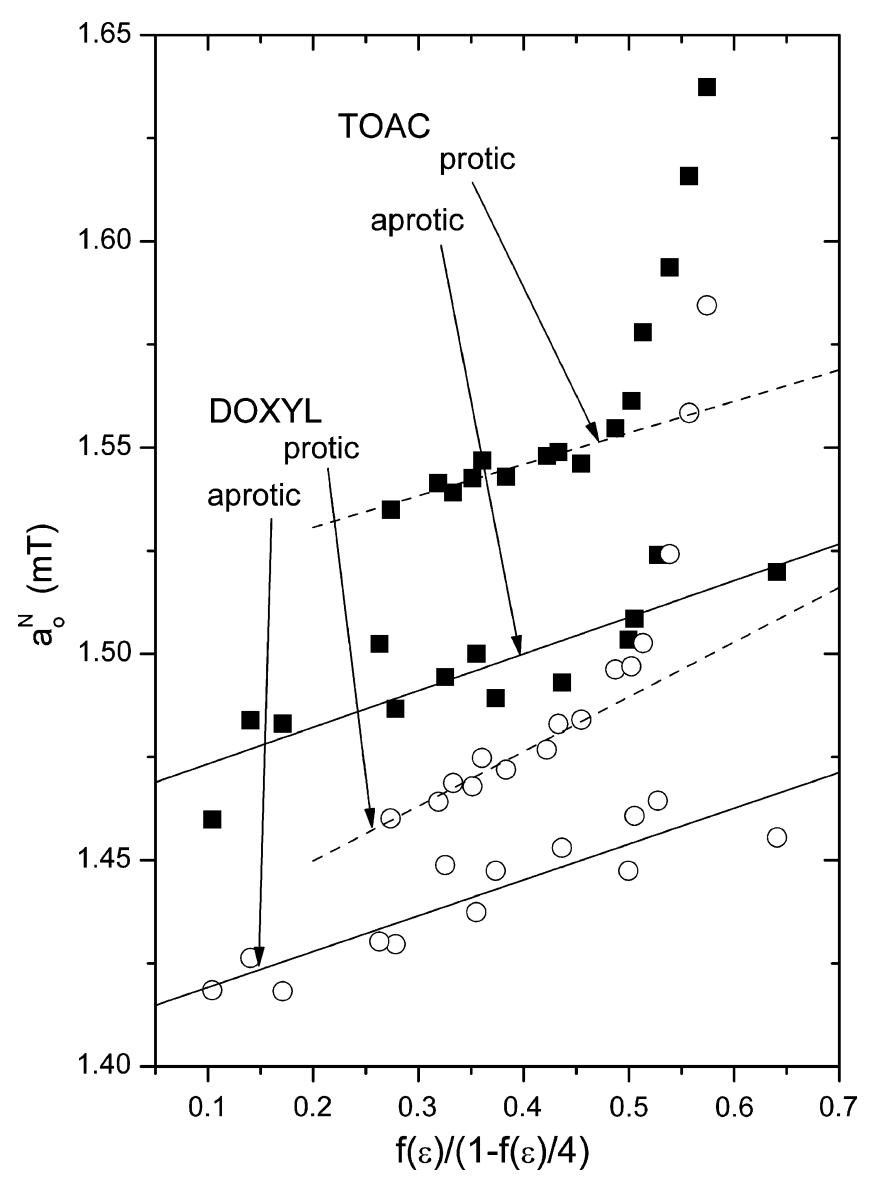

Fig. 2. Dependence of the isotropic hyperfine coupling, $a_{\mathrm{o}}^{\mathrm{N}}$, on solvent polarity, $f\left(\varepsilon_{\mathrm{r}}\right) /\left(1-\frac{1}{4} f\left(\varepsilon_{\mathrm{r}}\right)\right) \quad$ with $f\left(\varepsilon_{\mathrm{r}}\right)=3 \varepsilon_{\mathrm{r}} \ln \varepsilon_{\mathrm{r}} /\left(\varepsilon_{\mathrm{r}} \ln \varepsilon_{\mathrm{r}}-\varepsilon_{\mathrm{r}}+1\right)-6 /$ $\ln \varepsilon_{\mathrm{r}}-2$, for TOAC (squares) and DOXYL (circles) spin labels. Measurements are made at $20^{\circ} \mathrm{C}\left(30^{\circ} \mathrm{C}\right.$ for more viscous solvents) using FmocTOAC-Aib-OMe (TOAC) or 12-SASL (DOXYL). Solid lines are linear regressions to data from aprotic solvents, and dashed lines are linear regressions for a limited range of data from the more apolar protic solvents.

dence on $f\left(\varepsilon_{\mathrm{r}}\right) /\left(1-\frac{1}{4} f\left(\varepsilon_{\mathrm{r}}\right)\right)$ that is similar to that found here for the TOAC and DOXYL spin labels (data not shown). The corresponding slopes and intercepts obtained from linear regressions to the data for MTSSL and MTSSL/ $\beta-S H$ are listed in Table 1. Data for DTBN [1] are included additionally in Table 1 for comparison purposes. These correspond to a much wider range of aprotic solvents than used with MTSSL.

The relative values of $K_{v}$ in Table 1 presumably reflect the relative sizes of the different nitroxides, in particular that the largest value is obtained for the smallest nitroxide, DTBN (cf. Eq. (6)). Taking $\left(Q_{\mathrm{N}}-Q_{\mathrm{NO}}\right)=2.04 \mathrm{mT}$ [37], $p=3$ Debye $\left(10^{-29} \mathrm{Cm}\right)[40]$ and $C_{1}=1.9 \times 10^{-11} \mathrm{~V}^{-1} \mathrm{~m}$ (see Section 2.1), the following effective molecular radii are obtained from the values of $K_{v}$ for aprotic solvents by using Eq. (6): $r_{\text {eff }}=0.31 \pm 0.01,0.35 \pm 0.10$ and $0.38 \pm 0.07 \mathrm{~nm}$ for DTBN, MTSSL and MTSSL/ $\beta-\mathrm{SH}$, respectively. For the TOAC and DOXYL nitroxides an effective radius of $0.34 \pm 0.02 \mathrm{~nm}$ is obtained for each. Clearly, in these latter two cases, the effective values must 
Table 1

Dependence of isotropic nitrogen hyperfine coupling, $a_{\mathrm{o}}^{\mathrm{N}}$, on solvent polarity, $f\left(\varepsilon_{\mathrm{r}}\right) /\left(1-\frac{1}{4} f\left(\varepsilon_{\mathrm{r}}\right)\right)$, in aprotic and protic media according to Eqs. (4) and (5)

\begin{tabular}{|c|c|c|c|c|}
\hline \multirow[t]{2}{*}{ Spin label } & \multicolumn{2}{|c|}{$K_{v} \times 10^{2}(\mathrm{mT})$} & \multicolumn{2}{|l|}{$a_{\mathrm{o}}^{\varepsilon=1}(\mathrm{mT})$} \\
\hline & Aprotic & Protic & Aprotic & Protic \\
\hline \multirow[t]{2}{*}{ TOAC } & $8.9 \pm 1.6$ & $7.6 \pm 1.2$ & $1.464 \pm 0.006$ & $1.515 \pm 0.005$ \\
\hline & $(7.1 \pm 2.0$ & $8.7 \pm 2.6$ & $1.464 \pm 0.008$ & $1.500 \pm 0.010)^{\mathrm{a}}$ \\
\hline DOXYL & $8.7 \pm 1.2$ & $13.2 \pm 1.4$ & $1.410 \pm 0.005$ & $1.423 \pm 0.005$ \\
\hline MTSSL $^{\mathrm{b}}$ & $8.1 \pm 5.5$ & $9.1 \pm 0.9$ & $1.421 \pm 0.017$ & $1.461 \pm 0.004$ \\
\hline MTSSL $/ \beta-\mathrm{SH}^{\mathrm{b}}$ & $6.3 \pm 3.1$ & $10.4 \pm 0.2$ & $1.437 \pm 0.010$ & $1.467 \pm 0.001$ \\
\hline $\mathrm{DTBN}^{\mathrm{c}}$ & $11.5 \pm 1.1$ & $14.5 \pm 2.1$ & $1.509 \pm 0.004$ & $1.539 \pm 0.008$ \\
\hline
\end{tabular}

${ }^{\text {a }}$ Standard values are for Fmoc-TOAC-Aib-OMe; those in parentheses are for Fmoc-(Aib) ${ }_{2}$-TOAC-Aib-OMe, which has a more limited range of solubility.

${ }^{\mathrm{b}}$ Deduced from data in Owenius et al. [25].

${ }^{\mathrm{c}}$ Deduced from data in Griffith et al. [1].

correspond to only a segment of the entire spin-labelled molecule. Effective radii estimated from the group contributions and decrements to the molecular volume that are given by Bondi [41] are: $r_{\text {eff }}=\left(3 V_{w} / 4 \pi\right)^{1 / 3}=0.341,0.385$ and $0.386 \mathrm{~nm}$ for DTBN, MTSSL and MTSSL/ $\beta-\mathrm{SH}$, respectively. These are in qualitative accord with the values deduced from Table 1 and Eq. (6). For TOAC, the effective radius deduced from the molecular volume of the TOAC ring alone is $r_{\text {eff }}=0.335 \mathrm{~nm}$, which is close to the effective experimental value. The DOXYL unit has a considerably smaller molecular volume; including two methylene groups on either side of the point of chain attachment is needed to bring the effective radius, $r_{\text {eff }}=0.341 \mathrm{~nm}$, close to that deduced from Eq. (6).

Note that the effective values of molecular radii deduced from hyperfine couplings depend not only on the values assumed for $Q_{\mathrm{N}}-Q_{\mathrm{NO}}$ and $C_{1}$, but also on the model assumed for the reaction field (see e.g., [31]). For instance, effective molecular interaction radii deduced from values of $K_{v}$ corresponding to the Onsager model are systematically larger than those obtained by using Eq. (4). Therefore, reliance should be placed only on relative values and not on the absolute values deduced from Eqs. (5) and (6). Interestingly, density functional (DFT) calculations for the ring moiety of the MTSSL spin-label embedded in a homogeneous dielectric [25] yield results consistent with the Onsager model with $K_{v}=(13.5 \pm 0.1) \times 10^{-2} \mathrm{mT}$. This corresponds to an effective molecular radius of $r_{\text {eff }}=0.325 \mathrm{~nm}$ (with the above values of $Q_{\mathrm{N}}-Q_{\mathrm{NO}}$ and $C_{1}$ ), and that deduced from group volume contributions of the ring is $r_{\mathrm{eff}}=0.327 \mathrm{~nm}$.

These experimental comparisons suggest that the values assumed in applying Eq. (6) are not unreasonable. They imply that an electric field of strength $E_{x}=10^{9} \mathrm{~V} / \mathrm{m}$ produces a change in isotropic hyperfine coupling of $\Delta a_{\mathrm{o}}^{\mathrm{N}}=0.04 \mathrm{mT}$. This lies close to estimates made previously [1,28]. From Table 1 and the molecular volumes of DTBN, MTSSL, MTSSL/ $\beta-S H$ and the peptide moiety of TOAC, the mean experimental polarity sensitivity of the isotropic hyperfine coupling is given by: $K_{v}=(4.21 \pm$ $0.39) \times 10^{-3} \mathrm{mT} \mathrm{nm}^{3} \times\left(1 / r_{\mathrm{eff}}^{3}\right)$. This relation could prove useful for prediction of the polarity dependence of other nitroxides.

\subsection{Dependence of $a_{\mathrm{o}}^{\mathrm{N}}$ on hydrogen bonding}

The leveling-off of the polarity dependence for the more apolar protic solvents in Fig. 2 suggests that the bulkier alcohol molecules are less efficient at forming hydrogen bonds. It is expected that the fraction, $f_{\mathrm{h}}$, of hydrogenbonded nitroxides (cf. Eq. (7)) will depend on the concentration of proton-donor- $\mathrm{OH}$ groups in the different solvents. The latter is determined from the densities and molecular weights, together with additional data for ethanol-water mixtures from Mukerjee et al. [42]. Fig. 3A shows the dependence of $a_{\mathrm{o}}^{\mathrm{N}}$ on molar concentration, $[\mathrm{OH}]$, of hydroxyl groups in the alkanol solvents and their mixtures with water. An approximately linear dependence is obtained for each spin label that extrapolates to a value of $a_{\mathrm{o}, \mathrm{o}}^{\mathrm{N}} \approx 1.524 \pm 0.003$ and $1.449 \pm 0.003 \mathrm{mT}$ for the nonhydrogen-bonded TOAC and DOXYL nitroxides, respectively. These values of the hyperfine coupling are close to those of $1.51 \pm 0.02$ and $1.45 \pm 0.01 \mathrm{mT}$ for TOAC and

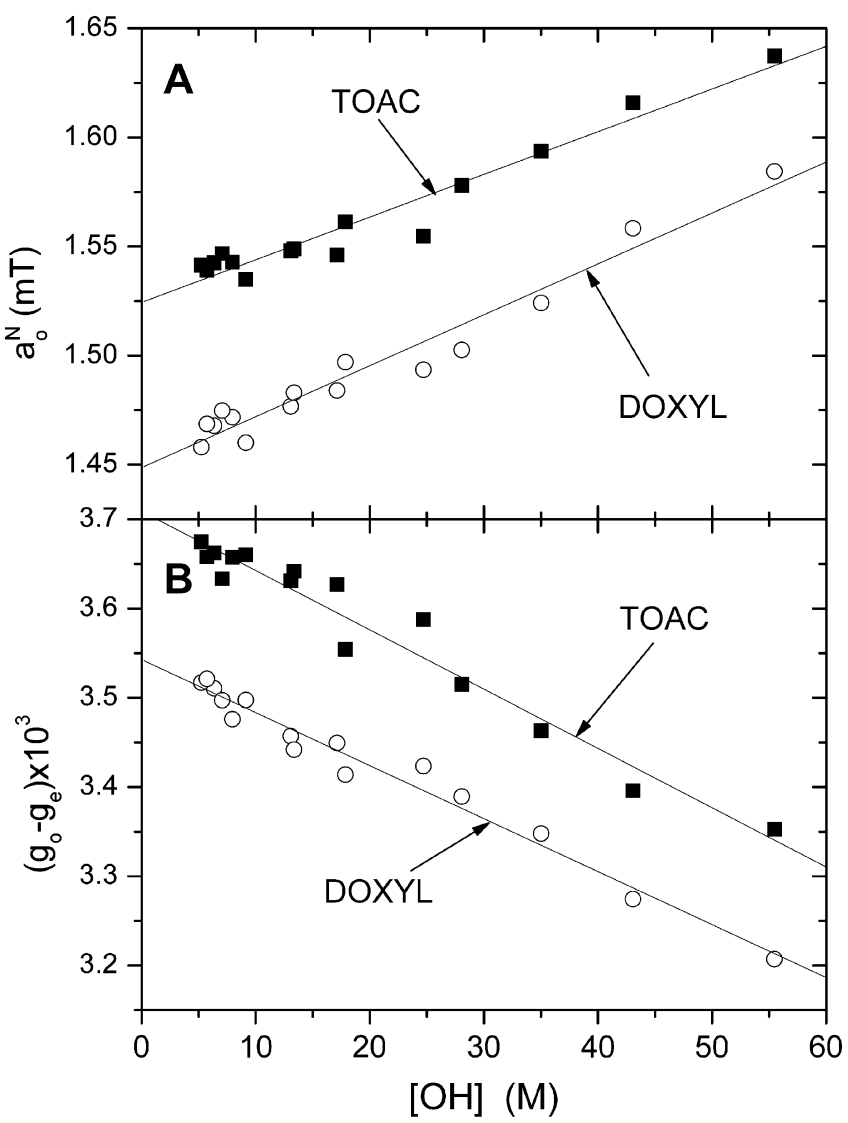

Fig. 3. Dependence of (A) the isotropic hyperfine coupling, $a_{\mathrm{o}}^{\mathrm{N}}$, and (B) the isotropic $g$-values, $g_{\mathrm{o}}$, on concentration, $[\mathrm{OH}]$, of hydroxyl protondonor groups in alkanols and their mixtures with water, for TOAC (squares) and DOXYL (circles) spin labels. The isotropic $g$-values are given relative to the free-electron $g$-value, $g_{\mathrm{e}}=2.002319$. Solid lines are linear regressions. 
DOXYL, respectively, in an aprotic solvent of polarity $f\left(\varepsilon_{\mathrm{r}}\right) /\left(1-\frac{1}{4} f\left(\varepsilon_{\mathrm{r}}\right)\right) \approx 0.5$ that are predicted from the data in Table 1.

The gradients of the dependences on $[\mathrm{OH}]$ concentration in Fig. $3 \mathrm{~A}$ are $\partial a_{\mathrm{o}}^{\mathrm{N}} / \partial[\mathrm{OH}]=(2.0 \pm 0.1) \times 10^{-3}$ and $(2.3 \pm 0.1) \times 10^{-3} \mathrm{mT} \mathrm{M}^{-1}$ for TOAC and DOXYL nitroxides, respectively. Similar values of $(2.2 \pm 0.2) \times$ $10^{-3} \mathrm{mT} \mathrm{M}^{-1}$ are also obtained for MTSSL from the data of Owenius et al. [25]. DFT calculations by Owenius et al. [25] for MTSSL predict a value of $a_{\mathrm{o}, \mathrm{h}}-a_{\mathrm{o}, \mathrm{o}} \approx 0.01$ and $0.07 \mathrm{mT}$ for one and two hydrogen bonds, respectively. This implies that $K_{A, \mathrm{~h}} \sim 0.03-0.2 \mathrm{M}^{-1}$, where $f_{\mathrm{h}}=K_{A, \mathrm{~h}}[\mathrm{OH}]$ in Eq. (7). Interpreted as an association constant, the standard state to which $K_{A \text {,h }}$ refers is that of a pure hydrogen-bonding solvent. Much higher association constants for hydrogen bonding are expected and found with nitroxides as acceptors in aprotic media $[34,35]$.

\subsection{Correlation of TOAC and MTSSL with DOXYL spin labels}

Fig. 4 (squares) shows the correlation between the isotropic hyperfine couplings of Fmoc-TOAC-Aib-OMe (TOAC) and those of 12-SASL (DOXYL), in the same solvents. The data fall into two groups, that with larger values of $a_{\mathrm{o}}^{\mathrm{N}}$ is for protic solvents and that with smaller $a_{\mathrm{o}}^{\mathrm{N}}$ is for aprotic solvents. From Eq. (5) it is expected that the isotropic hyperfine splitting for TOAC in aprotic solvents is linearly related to that of a DOXYL nitroxide in the same solvent. In protic solvents, a linear dependence is expected also-from Eq. (7). In general, the gradient and intercept of the linear dependences between the hyperfine couplings

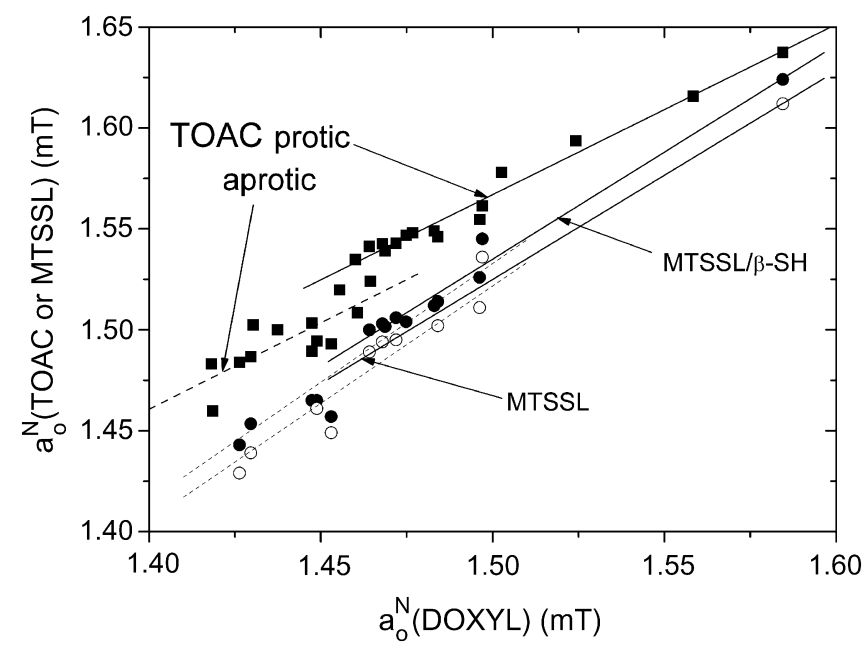

Fig. 4. Correlation of isotropic hyperfine coupling constants, $a_{\mathrm{o}}^{\mathrm{N}}$, of TOAC (squares) and MTSSL (circles) spin labels with those of DOXYL spin labels in the same solvents. Data for free MTSSL (open circles) and MTSSL/ $\beta$-SH (solid circles) are from Owenius et al. [25]. Solid lines are linear regressions to data from protic solvents. Dotted lines are linear regressions to the data for TOAC in aprotic solvents, and to all data, in the case of MTSSL. of the two nitroxides will differ between protic and aprotic solvents.

In Fig. 4, the uppermost solid and dashed lines represent linear regressions to the data for TOAC in protic and aprotic solvents, respectively. For protic solvents, the slope and intercept of the linear correlation are $0.844 \pm 0.037$ and $0.302 \pm 0.055 \mathrm{mT}$, respectively $(R=0.988)$. For aprotic solvents, the scatter is considerably greater although the slope is similar. It is seen from Fig. 2 that the slopes of the polarity dependences of the hyperfine couplings for TOAC and DOXYL spin labels in aprotic solvents are almost equal. Hence, a useful relation that can be deduced from the intercepts, $a_{\mathrm{o}}^{\varepsilon=1}$, in Table 1 is that $a_{\mathrm{o}}^{\mathrm{N}}(\mathrm{TOAC}) \approx a_{\mathrm{o}}^{\mathrm{N}}(\mathrm{DOXYL})+0.05 \mathrm{mT}$. This approximation for aprotic solvents has greater precision than can be deduced from the linear regression in Fig. 4.

Corresponding data for MTSSL and MTSSL/ $\beta$-SH from Owenius et al. [25] are also given in Fig. 4 (circles), for solvents that are in common with those used in the present study. The number of aprotic solvents is relatively small, although a clear distinction can still be seen. Linear regressions for the datasets from the protic solvents in common yield slopes of $1.060 \pm 0.055$ and $1.033 \pm 0.082$ for MTSSL coupled to $\beta$-mercaptoethanol and for free MTSSL, respectively. Correspondingly, the intercepts for protic solvents are $-0.06 \pm 0.08$ and $-0.02 \pm 0.12 \mathrm{mT}$, respectively. For purely illustrative purposes, the lower dashed lines in Fig. 4 represent part of linear regressions to the entire data sets for MTSSL and MTSSL/ $\beta-\mathrm{SH}$. They serve to indicate that the hyperfine couplings in aprotic solvents lie significantly below the linear regressions for protic solvents.

\subsection{Calibration of penetration depth for TOAC and MTSSL in membranes, using DOXYL-labelled lipids}

Dotted lines in Fig. 5 show the dependence on the chain position, $n$, of the isotropic hyperfine coupling, $a_{\mathrm{o}}^{\mathrm{N}}$, for the $n$-PCSL (1-acyl-2-[n-(4,4-dimethyl-oxazolidin-1-oxyl)]stearoyl-sn-glycero-3-phosphocholine) spin-labelled phosphatidylcholines in fluid-phase lipid bilayers of dipalmitoyl phosphatidylcholine (DPPC) or of a $1: 1 \mathrm{~mol} / \mathrm{mol}$ mixture of DPPC with cholesterol. As established experimentally for a range of different lipids, the transmembrane profile of $a_{\mathrm{o}}^{\mathrm{N}}$ follows the sigmoidal Boltzmann two-phase distribution [9]:

$a_{\mathrm{o}}^{\mathrm{N}}=\frac{a_{\mathrm{o}, 1}^{\mathrm{N}}-a_{\mathrm{o}, 2}^{\mathrm{N}}}{1+\exp \left(\left(n-n_{\mathrm{o}}\right) / \lambda\right)}+a_{\mathrm{o}, 2}^{\mathrm{N}}$

where $a_{\mathrm{o}, 1}^{\mathrm{N}}$ and $a_{\mathrm{o}, 2}^{\mathrm{N}}$ are the limiting values of $a_{\mathrm{o}}^{\mathrm{N}}$ at the polar headgroup and terminal methyl ends of the lipid chain and $\lambda$ is an exponential decay length (see also $[43,44])$. The correlations established here between the polarity dependence of the TOAC or MTSSL spin labels and that of the DOXYL fatty acid or phospholipid spin labels allow one to develop calibrations for the positional dependence of the 


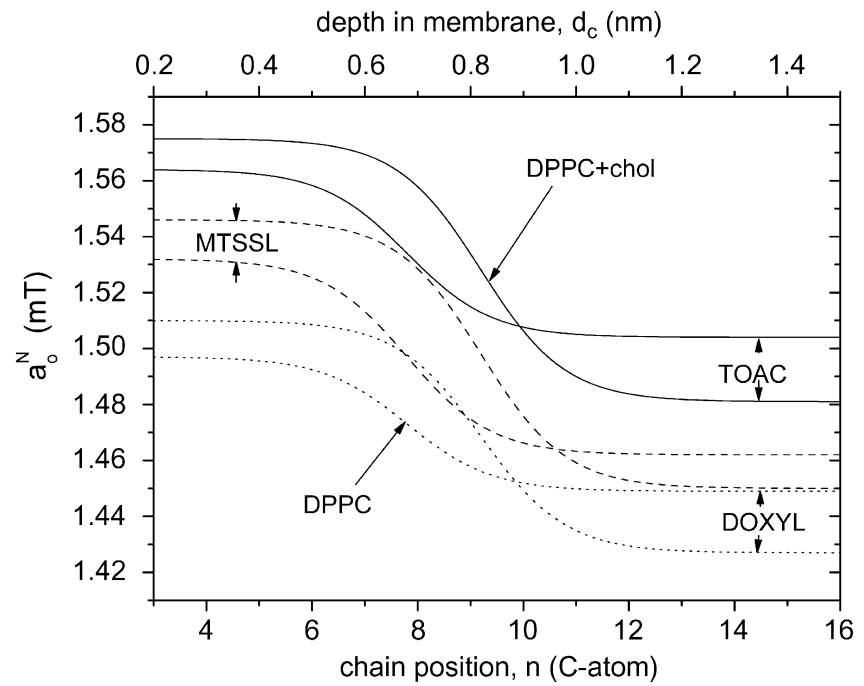

Fig. 5. Predicted dependence of the isotropic hyperfine coupling, $a_{\mathrm{o}}^{\mathrm{N}}$, on depth in fluid DPPC or DPPC $+50 \mathrm{~mol} \%$ cholesterol (chol), bilayer membranes, for TOAC (solid lines) and MTSSL (dashed lines) spin labels. Calibration is deduced from $n$-PCSL spin-labelled phospholipid chains (dotted lines) in membranes [9], and the correlations of TOAC and MTSSL spin labels with DOXYL spin labels. $n$ is the DOXYL-labelled Catom in the $s n-2$ chain of $n$-PCSL, and $d_{\mathrm{c}}$ is the estimated distance from the carbonyl group $(n=1)$ (see [47]).

isotropic hyperfine couplings of TOAC and MTSSL spin labels in membranes. This calibration is carried out for fluid bilayers of DPPC and DPPC $+50 \mathrm{~mol} \%$ cholesterol in Fig. 5.

Correlations for protic solvents are used in correcting values of $a_{\mathrm{o}, 1}^{\mathrm{N}}$ and those for aprotic solvents in correcting $a_{\mathrm{o}, 2}^{\mathrm{N}}$, in Fig. 5. This distinction is justified both by $\mathrm{D}_{2} \mathrm{O}-$ ESEEM experiments on water penetration $[45,46]$, and by $g_{x x}$-inhomogeneities arising from hydrogen bonding that are observed in high-field EPR [10]. To a good approximation, the depth dependence in phospholipid membranes other than those in Fig. 5 can be obtained from Eq. (14), with the appropriate parameters deduced from the $n$-PCSL spin labels [9], except that $a_{\mathrm{o}, 1}^{\mathrm{N}}$ is increased by $0.065 \mathrm{mT}$ for TOAC and by $0.036 \mathrm{mT}$ for MTSSL/ $\beta-\mathrm{SH}$, and $a_{\mathrm{o}, 2}^{\mathrm{N}}$ is correspondingly increased by $0.054 \mathrm{mT}$ for $\mathrm{TOAC}$ and by $0.021 \mathrm{mT}$ for MTSSL/ $\beta$-SH.

Finally, a correlation between the chain position and depth in the membrane can be established from the results of X-ray diffraction measurements [47]. This correlation is performed in Fig. 5, where the upper abscissa is the estimated distance, $d_{c}$, from the chain carbonyl group $(n=1)$ in fluid DPPC membranes. This corresponds to a carbonyl to carbonyl separation across the DPPC bilayer of approximately $3.0 \mathrm{~nm}$, in reasonable agreement with experiment.

\subsection{Anisotropic hyperfine couplings}

Spectra of Fmoc-TOAC-Aib-OMe in the different solvents were recorded in the frozen state at $77 \mathrm{~K}$. In certain cases, e.g., in $n$-hexane and in water, the spin-labelled pep- tide was excluded completely from the frozen matrix. In many other cases, the peptide was only partly incorporated and the spectra evidenced strong spin-spin interactions. As a result, there was very considerable scatter in the correlation of the anisotropic hyperfine coupling $A_{z z}$ measured from powder patterns at low temperature with the isotropic hyperfine couplings $a_{\mathrm{o}}^{\mathrm{N}}$ measured in fluid solutions. An additional consideration, for such correlations, is that the temperature dependence of the dielectric constant differs greatly between polar and apolar media [48]. A linear correlation between $A_{z z}$ and $a_{\mathrm{o}}^{\mathrm{N}}$ could not be established for the TOAC spin-label. Under these circumstances, the most useful parameter that can be derived for TOAC is the mean ratio $A_{z z} / a_{\mathrm{o}}^{\mathrm{N}}=2.32 \pm 0.07$ (mean $\pm \mathrm{SD}, N=27$ ). If it is assumed that $Q_{\mathrm{NO}} \approx 0$ in Eq. (9), this ratio implies an upper estimate of $Q_{\mathrm{N}} /\left|T_{\perp . o}^{\mathrm{d}}\right| \approx 1.52 \pm 0.08$, i.e., $Q_{\mathrm{N}} \approx 72 \pm$ $4 \mathrm{MHz}(2.58 \pm 0.13 \mathrm{mT})$.

Similar considerations apply to the spectra of the DOXYL-labelled fatty acid 12-SASL, although somewhat less acute. The mean ratio of the anisotropic to isotropic couplings for the different solvents is $A_{z z} / a_{\mathrm{o}}^{\mathrm{N}}=2.33 \pm 0.05$ (mean $\pm \mathrm{SD}, N=26$ ). This value for the DOXYL nitroxide is practically the same as that for the TOAC nitroxide and, correspondingly, the estimate for $Q_{\mathrm{N}}$ is similar, to the above degree of approximation. Griffith et al. [1] demonstrated an approximately linear relation between $A_{z z}$ and $a_{\mathrm{o}}^{\mathrm{N}}$ for DOXYL spin-labelled stearic acids or their methyl esters (depending on solvent) in a number of glass-forming media. Linear regression to this data yields $A_{z z}=$ $(2.19 \pm 0.27) \times a_{\mathrm{o}}^{\mathrm{N}}+(0.14 \pm 0.39) \mathrm{mT}$, implying from Eq. (9) that $Q_{\mathrm{NO}} \approx 0$. For comparison with the present results, the mean ratio is $A_{z z} / a_{\mathrm{o}}^{\mathrm{N}}=2.29 \pm 0.02$ (mean $\pm \mathrm{SD}$, $N=7)$, which corresponds to $Q_{\mathrm{N}} \approx 74 \pm 1 \mathrm{MHz}(2.64 \pm$ $0.04 \mathrm{mT}$ ).

Considerable scatter in the solvent dependence of $A_{z z}$ was also found for MTSSL spin labels by Owenius et al. [25]. Mean ratios of $A_{z z} / a_{\mathrm{o}}^{\mathrm{N}}=2.34 \pm 0.04$ (mean $\pm \mathrm{SD}$, $N=8,11)$ are obtained from the data for both MTSSL and MTSSL/ $\beta-\mathrm{SH}$. Less scatter was obtained with measurements of $A_{z z}$ at higher frequency ( $94 \mathrm{GHz}$, [25]) from which an approximate linear correlation is obtained with a mean value of $A_{z z} / a_{\mathrm{o}}^{\mathrm{N}}=2.36 \pm 0.02 \quad$ (mean $\pm \mathrm{SD}$, $N=7$ ), which corresponds to $Q_{\mathrm{N}} \approx 70 \pm 1 \mathrm{MHz}$ $(2.50 \pm 0.04 \mathrm{mT})$ for MTSSL/ $\beta-\mathrm{SH}$.

\subsection{Polarity dependence of isotropic g-values}

Fig. 6 shows the dependence of the isotropic $g$-value, $g_{\mathrm{o}}$, on the strength of the polarisation reaction field of the solvent, $f\left(\varepsilon_{\mathrm{r}}\right) /\left(1-\frac{1}{4} f\left(\varepsilon_{\mathrm{r}}\right)\right)$, for the TOAC and DOXYL spin labels. As with the isotropic hyperfine splitting (see Fig. 2), the data again fall into two groups, which is particularly evident for the TOAC peptide (squares). The values of $g_{\mathrm{o}}$ for aprotic solvents decrease approximately linearly with polarity of the solvent. Solid lines in Fig. 6 are linear regressions to the data for aprotic solvents. Values of the gradient, $K_{v, g}$, and intercept, $g_{\mathrm{o}}^{\varepsilon=1}$, according to Eq. (12) 


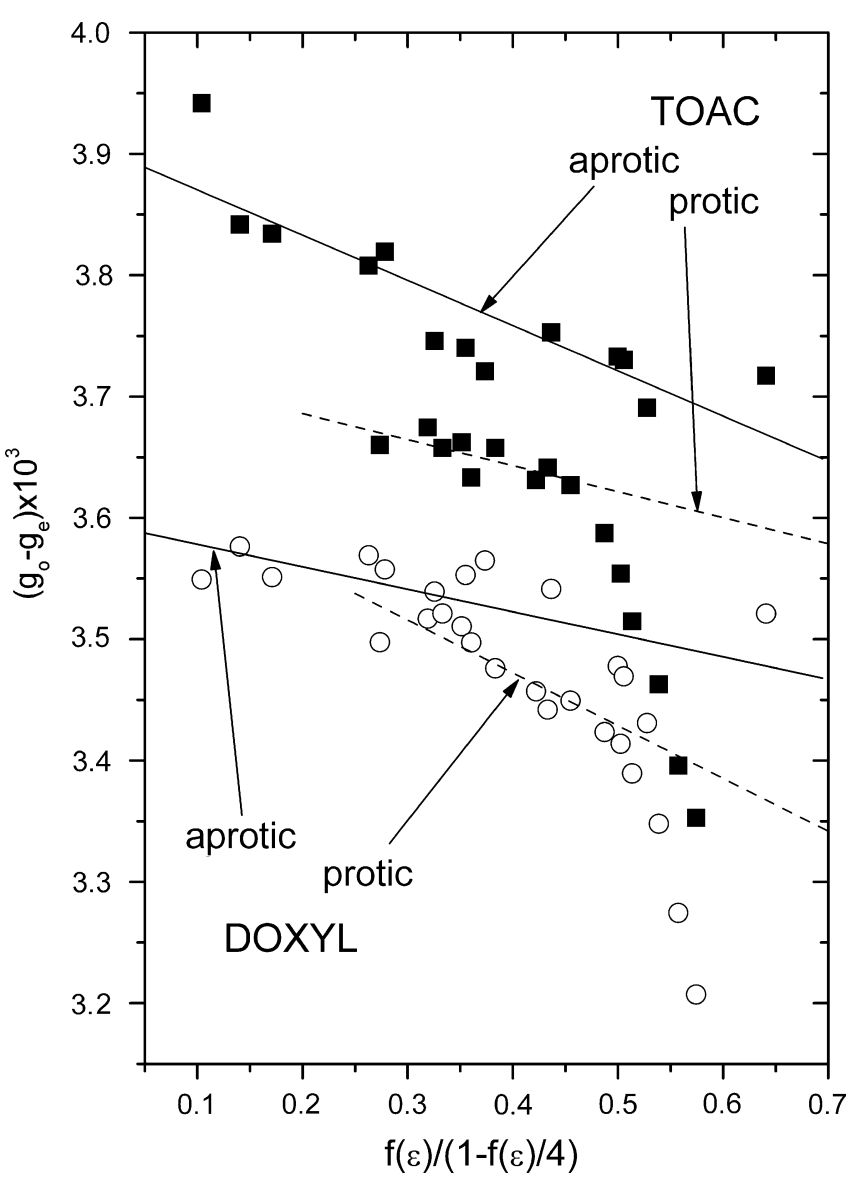

Fig. 6. Dependence of the isotropic $g$-value, $g_{\mathrm{o}}$, on solvent polarity, $f\left(\varepsilon_{\mathrm{r}}\right) /\left(1-\frac{1}{4} f\left(\varepsilon_{\mathrm{r}}\right)\right)$ with $f\left(\varepsilon_{\mathrm{r}}\right)=3 \varepsilon_{\mathrm{r}} \ln \varepsilon_{r} /\left(\varepsilon_{\mathrm{r}} \ln \varepsilon_{r}-\varepsilon_{\mathrm{r}}+1\right)-6 / \ln \varepsilon_{r}-2$, for TOAC (squares) and DOXYL (circles) spin labels. Measurements are made at $20^{\circ} \mathrm{C}\left(30^{\circ} \mathrm{C}\right.$ for more viscous solvents) using Fmoc-TOAC-AibOMe (TOAC) or 12-SASL (DOXYL) spin labels. The ordinate is expressed relative to the free-electron $g$-value, $g_{\mathrm{e}}=2.002319$. Solid lines are linear regressions to data from aprotic solvents, and dashed lines are linear regressions for a limited range of data from the more apolar protic solvents.

are given in Table 2. Corresponding results for MTSSL and DTBN, deduced from data in the literature $[1,25]$ are also included in Table 2.

The isotropic $g$-values decrease greatly in strongly $\mathrm{H}$-bonding solvents. However, as for the isotropic hyperfine couplings, the isotropic $g$-values of the more apolar protic solvents depend approximately linearly on $f\left(\varepsilon_{\mathrm{r}}\right) /\left(1-\frac{1}{4} f\left(\varepsilon_{\mathrm{r}}\right)\right)$, with a gradient that is similar to that for the aprotic solvents. This feature is evident for the TOAC nitroxide (see dashed line in Fig. 6), but less so for the DOXYL nitroxide. In general, the polarity dependence of $g_{\mathrm{o}}$ for the DOXYL nitroxide in apolar solvents is less pronounced than that for the TOAC nitroxide.

As for the hyperfine couplings, the isotropic $g$-values depend approximately linearly on the concentration of hydroxyl groups in hydrogen-bonding solvents. The corresponding data are given in Fig. 3B. The extrapolated values at $[\mathrm{OH}]=0$ are $\left(g_{\mathrm{o}, \mathrm{o}}-g_{\mathrm{e}}\right) \approx(3.709 \pm 0.010) \times 10^{-3}$ and $(3.543 \pm 0.007) \times 10^{-3}$ for TOAC and DOXYL nitroxides,
Table 2

Dependence of isotropic $g$-values, $g_{o}$, on solvent polarity, $f\left(\varepsilon_{\mathrm{r}}\right) /\left(1-\frac{1}{4} f\left(\varepsilon_{\mathrm{r}}\right)\right)$, in aprotic media according to Eqs. (4) and (12), and correlation of $g_{\mathrm{o}}$ with isotropic hyperfine coupling, $a_{\mathrm{o}}^{\mathrm{N}}$, for protic media

\begin{tabular}{llll}
\hline Spin label & $K_{v, g} \times 10^{3}$ & $\left(g_{\mathrm{o}}^{\varepsilon=1}-g_{\mathrm{e}}\right) \times 10^{3}$ & $\partial g_{\mathrm{o}} / \partial a_{\mathrm{o}}^{\mathrm{N}}\left(\mathrm{T}^{-1}\right)$ \\
\hline TOAC & $-0.37 \pm 0.06$ & $3.91 \pm 0.02$ & $-3.33 \pm 0.14$ \\
DOXYL & $-0.19 \pm 0.06$ & $3.60 \pm 0.02$ & $-2.52 \pm 0.11$ \\
MTSSL $^{\mathrm{a}}$ & $-0.22 \pm 0.09$ & $3.65 \pm 0.03$ & $-2.52 \pm 0.12$ \\
${\text { MTSSL/ } \beta-S^{\mathrm{a}}}^{\mathrm{a}}$ & $-0.36 \pm 0.15$ & $3.69 \pm 0.04$ & $-2.46 \pm 0.10$ \\
DTBN $^{\mathrm{b}}$ & $-0.38 \pm 0.05$ & $3.84 \pm 0.02$ & $-2.30 \pm 0.09$ \\
DFT $^{\mathrm{c}}$ & & & $-2.1,-2.2$ \\
\hline
\end{tabular}

${ }^{\text {a }}$ Deduced from data in Owenius et al. [25].

${ }^{\mathrm{b}}$ Deduced from data in Griffith et al. [1].

${ }^{\mathrm{c}}$ Density functional calculations. Data from Owenius et al. [25]. Contributions solely from hydrogen bonding: 1 and 2 hydrogen-bonds, respectively.

respectively. Again these values correspond reasonably closely to those estimated for aprotic solvents of polarity $f\left(\varepsilon_{\mathrm{r}}\right) /\left(1-\frac{1}{4} f\left(\varepsilon_{\mathrm{r}}\right)\right) \approx 0.5, \quad$ viz. $\quad(3.72 \pm 0.06) \times 10^{-3} \quad$ and $(3.50 \pm 0.05) \times 10^{-3}$ for TOAC and DOXYL, respectively, as deduced from Table 2 . The gradients of the linear regressions in Fig. 3B are: $\partial g_{0} / \partial[\mathrm{OH}]=-(6.6 \pm 0.4) \times 10^{-6}$ and $-(5.9 \pm 0.3) \times 10^{-6} \mathrm{M}^{-1}$ for TOAC and DOXYL, respectively. Similarly, for $\mathrm{MTSSL} / \beta-\mathrm{SH}: \quad \partial g_{\mathrm{o}} / \partial[\mathrm{OH}]=$ $-(5.5 \pm 0.5) \times 10^{-6}$, and $-(5.7 \pm 0.7) \times 10^{-6} \mathrm{M}^{-1}$ for MTSSL, from the data of Owenius et al. [25]; and also it was estimated that $\left(g_{\mathrm{o}, \mathrm{h}}-g_{\mathrm{o}, \mathrm{o}}\right) \approx-1.7 \times 10^{-4}$ and $-3.2 \times 10^{-4}$ for one and two hydrogen bonds, respectively, from DFT calculations. This gives $K_{A, \mathrm{~h}} \sim 0.02-0.04 \mathrm{M}^{-1}$, in approximate agreement with the corresponding estimate from the hyperfine couplings. Clearly, these estimates depend, among other things, upon the reliability of the DFT calculations.

\subsection{Correlation between polarity dependencies of $g_{o}$ and $a_{o}$}

Fig. 7 shows the correlation between the isotropic $g$-values and isotropic hyperfine couplings in a given solvent, for the nitroxides TOAC, DOXYL and MTSSL. From Eqs. (7) and (13), $g_{\mathrm{o}}$ is predicted to depend linearly on $a_{\mathrm{o}}^{\mathrm{N}}$ in protic solvents. Correspondingly, a linear dependence is also predicted from Eqs. (5) and (12) for aprotic solvents. In general, the gradients and intercepts are not expected to be the same in protic and aprotic solvents (cf. [2]). However, as found previously for DTBN and MTSSL $[1,25]$, there is little obvious distinction between the data for protic and aprotic solvents in Fig. 7. The straight lines in Fig. 7 represent linear regressions to the data from protic solvents, although these differ relatively little from linear regressions to the entire dataset. Values of the gradients of the linear regressions, $\partial g_{\mathrm{o}} / \partial a_{\mathrm{o}}^{\mathrm{N}}$, for protic solvents are included in Table 2.

With the possible exception of TOAC, the values of $\partial g_{\mathrm{o}} / \partial a_{\mathrm{o}}^{\mathrm{N}}$ in Table 2 are rather similar in magnitude. For comparison, a value of $\partial g_{\mathrm{o}} / \partial a_{\mathrm{o}}^{\mathrm{N}}=-2.3 \pm 0.4 \mathrm{~T}^{-1}$ was obtained for DOXYL-labelled lipid chains in phosphatidylcholine 


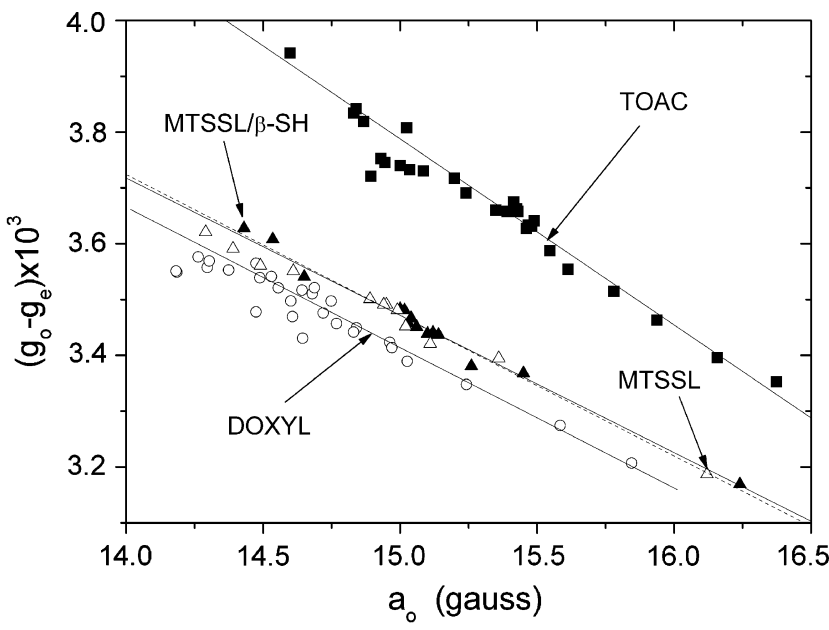

Fig. 7. Correlation of isotropic $g$-values, $g_{0}$, with isotropic hyperfine couplings, $a_{\mathrm{o}}^{\mathrm{N}}$, for TOAC (squares), MTSSL (triangles), and DOXYL (circles) spin labels, in the same solvents. The ordinate is expressed relative to the free-electron $g$-value, $g_{\mathrm{e}}=2.002319$. Data for free MTSSL (open triangles, dashed line) and MTSSL/ $\beta$-SH (solid triangles, solid line) are from Owenius et al. [25]. Lines are linear regressions to data from protic solvents.

bilayer membranes [10]. Predictions from DFT calculations ([25]; see Table 2) suggest that a large part of this gradient in strongly polar solvents is contributed by hydrogen bonding to the nitroxide. High-field EPR measurements reveal a steeper $\partial g_{x x} / \partial A_{z z}$ gradient in protic than in aprotic environments $[8,25]$, which is in line with this suggestion.

From Eqs. (1) and (11), it follows that changes in $g_{\mathrm{o}}$ and $a_{\mathrm{o}}^{\mathrm{N}}$ are related, via the dependence on spin density, by:

$\frac{\delta g_{\mathrm{o}}}{g_{\mathrm{o}}-g_{\mathrm{e}}}=-\frac{\rho_{\pi}^{\mathrm{N}}}{\rho_{\pi}^{\mathrm{O}}} \frac{\delta a_{\mathrm{o}}^{\mathrm{N}}}{a_{\mathrm{o}}^{\mathrm{N}}}-\frac{\delta \Delta E_{n \pi^{*}}}{\Delta E_{n \pi^{*}}}+\frac{\delta\left(C_{\mathrm{O}, y}^{(n)}\right)^{2}}{\left(C_{\mathrm{O}, y}^{(n)}\right)^{2}}+\frac{\delta g_{y y}}{g_{y y}}$

From Fig. 6, the maximum extent of $\delta g_{\mathrm{o}} /\left(g_{\mathrm{o}}-g_{\mathrm{e}}\right)$ is $\sim$ $-13 \%$, and that of $\delta a_{\mathrm{o}}^{\mathrm{N}} / a_{\mathrm{o}}^{\mathrm{N}}$ from Fig. 2 is $\sim 12 \%$. Because $\rho_{\pi}^{\mathrm{N}} \approx \rho_{\pi}^{\mathrm{O}}$ for nitroxides, the remaining terms on the right of Eq. (15) make a net contribution to $\delta g_{\mathrm{o}} /\left(g_{\mathrm{o}}-g_{\mathrm{e}}\right)$ of $\sim$ $-1 \%$. This analysis therefore also predicts an approximately linear relation between $g_{\mathrm{o}}$ and $a_{\mathrm{o}}$, with a slope that is given by:

$\frac{\partial g_{\mathrm{o}}}{\partial a_{\mathrm{o}}^{\mathrm{N}}} \approx-\left(\frac{g_{\mathrm{o}}-g_{\mathrm{e}}}{a_{\mathrm{o}}^{\mathrm{N}}}\right) \frac{\rho_{\pi}^{\mathrm{N}}}{1-\rho_{\pi}^{\mathrm{N}}}$

With the data in Fig. 7 and Table 2, the spin density in TOAC nitroxides is predicted by Eq. (16) to vary from $\rho_{\pi}^{\mathrm{N}}=0.53$ in $n$-hexane to $\rho_{\pi}^{\mathrm{N}}=0.61$ in water, and similarly from 0.49 to 0.56 , respectively, in MTSSL nitroxides, and from 0.47 to 0.55 in DOXYL nitroxides.

\section{Conclusions}

The above study of the factors determining the polarity dependence of nitroxide spin-label EPR spectra establishes the calibrations needed to transfer data on environmental polarity between different spin labels. This is especially important for transverse polarity profiles in biological membranes (see Section 3.4). The reaction field proposed by Block and Walker [32], rather than that of Onsager [29], gives a more reliable description of the polarity dependence in polar solvents [30] and thus provides a more sensitive correlation between spin labels in environments with a wider range of polarities.

When feasible, comparisons are best made with the isotropic hyperfine couplings. Practical considerations for the measurement of $a_{\mathrm{o}}^{\mathrm{N}}$ from anisotropic spin-label EPR spectra in biological systems are given in Refs. $[12,13,17,18]$. For aprotic environments, the necessary relations are established by a common effective dielectric constant. For protic solvents, on the other hand, connections are established by a common degree of hydrogen bonding. Illustrations of applications of these correlations are given in Section 3.4.

If it is not possible to determine the isotropic hyperfine couplings, $a_{\mathrm{o}}^{\mathrm{N}}$, reliably in the biological system, then resort must be made to the anisotropic hyperfine couplings, $A_{z z}$. These are obtained from the hyperfine splittings in rigidlimit spectra at low temperature. When values of $A_{z z}$ are to be used as a polarity index, they are best transferred to the polarity indices given here for isotropic couplings by means of the ratio $A_{z z} / a_{\mathrm{o}}^{\mathrm{N}}$. Values of this ratio are given for the different nitroxide spin labels in Section 3.5.

Finally, isotropic $g$-factors are valuable additions to characterisation of environmental polarity (see Sections 3.6 and 3.7). If the isotropic $g$-values cannot be measured reliably, then (unlike for hyperfine couplings) rigid-limit spectra at $9 \mathrm{GHz}$ are not helpful, because the $g_{z z}$-tensor element that can be extracted from such spectra is not sensitive to polarity [2]. Resort must then be made to high-frequency measurements of the $g_{x x}$-tensor element, which is particularly useful for diagnostics of protic environments $[8,39,49]$.

\section{Experimental}

Dipeptide Fmoc-TOAC-Aib-OMe (where Fmoc is fluoren-9-ylmethyloxycarbonyl, Aib is $\alpha$-aminoisobutyric acid, and $\mathrm{OMe}$ is methoxy) was synthesised in solution using Fmoc-TOAC-OH and either the 1-(3-dimethylaminopropyl)-3-ethylcarbodiimide (EDC)/7-aza-1-hydroxy-1,2, 3-benzotriazole (HOAt) [50], or the $N$-[(dimethylamino)$1 H$-1,2,3-triazolo[4,5- $b$ ]pyridin-1-yl-methylene]- $N$-methylmethanaminium hexafluorophosphate $N$-oxide (HATU) [50], C-activation method. Yield $80 \%$. M.p.: $174-175{ }^{\circ} \mathrm{C}$; IR (KBr): 3381, 1722, 1658, $1525 \mathrm{~cm}^{-1}$; MS (ESI-TOF): $[\mathrm{M}+\mathrm{H}]^{+} 537.2793$ for a calculated MW of 536.2760. The 12-SASL DOXYL-containing, stearic acid derivative [D,L2-(10-carboxydecyl)-2-hexyl-4,4-dimethyl-3-oxazolidinyl-1oxy] was synthesised according to Keana et al. [51].

The solvents used throughout (dielectric constants from the literature are given in parentheses) are (i) aprotic solvents: hexane (1.89), di- $n$-propylamine (2.9), $n$-butylamine (5.4), ethyl acetate (6.02), ethyl acetate saturated with water 
(10.6), acetone (20.7), $N, N$-dimethylformamide (36.7), acetonitrile (38.8), dimethyl sulphoxide (48.17), $N$-methyl propionamide (171.5); (ii) protic solvents: 2-methyl-2-butanol (5.82), 1-decanol (8.1), 1-octanol (10.34), 1-hexanol (13.3), 2-propanol (18.3), 1-propanol (20.1), ethanol (24.3), methanol (32.62), 1,2-ethanediol (37.7), ethanol/water 7:3 v/v (41.9), 1:1 v/v (54.0), 3:7 v/v (65.4), water (78.54). Some additional measurements were also made with the following solvents: toluene (2.379), methyl formate (8.5), pyridine (12.3), 1-heptanol (11.1) and 1-nonanol (9).

EPR spectra were recorded on a Varian Century-Line 9$\mathrm{GHz}$ spectrometer (Varian, Palo Alto, CA) with $100 \mathrm{kHz}$ field modulation. The static magnetic field was measured with a Bruker (Karlsruhe, Germany) ER 035M NMR magnetometer and the microwave frequency with a Hewlett Packard 5345A/5355A frequency counter. para-Benzosemiquinone in alkaline 1-butanol $(g=2.004665 \pm 0.000006$; $[52,53])$ and $\alpha, \alpha$-diphenyl- $\beta$-picrylhydrazyl $(g=2.00369 \pm$ 0.00003 ) were used as $g$-value standards. Measurements at low temperature were made with a quartz-finger immersion Dewar.

\section{Acknowledgement}

We thank Frau B. Angerstein for DOXYL spin label synthesis and expert technical assistance with EPR measurements.

\section{References}

[1] O.H. Griffith, P.J. Dehlinger, S.P. Van, Shape of the hydrophobic barrier of phospholipid bilayers. Evidence for water penetration in biological membranes, J. Membr. Biol. 15 (1974) 159-192.

[2] T. Kawamura, S. Matsunami, T. Yonezawa, Solvent effects on the $g$ value of di-t-butyl nitric oxide, Bull. Chem. Soc. Jpn. 40 (1967) 11111115 .

[3] D. Marsh, D. Kurad, V.A. Livshits, High-field electron spin resonance of spin labels in membranes, Chem. Phys. Lipids 116 (2002) 93-114.

[4] M. Plato, H.J. Steinhoff, C. Wegener, J.T. Törring, A. Savitsky, K. Möbius, Molecular orbital study of polarity and hydrogen bonding effects on the $g$ and hyperfine tensors of site directed NO spin labelled bacteriorhodopsin, Mol. Phys. 100 (2002) 3711-3721.

[5] P. Fretten, S.J. Morris, A. Watts, D. Marsh, Lipid-lipid and lipidprotein interactions in chromaffin granule membranes, Biochim. Biophys. Acta 598 (1980) 247-259.

[6] R.D. Pates, D. Marsh, Lipid mobility order in bovine rod outer segment disk membranes. A spin-label study of lipid-protein interactions, Biochemistry 26 (1987) 29-39.

[7] W.K. Subczynski, A. Wisniewska, J.J. Yin, J.S. Hyde, A. Kusumi, Hydrophobic barriers of lipid bilayer membranes formed by reduction of water penetration by alkyl chain unsaturation and cholesterol, Biochemistry 33 (1994) 7670-7681.

[8] H.J. Steinhoff, A. Savitsky, C. Wegener, M. Pfeiffer, M. Plato, K. Möbius, High-field EPR studies of the structure and conformational changes of site-directed spin labeled bacteriorhodopsin, Biochim. Biophys. Acta 1457 (2000) 253-262.

[9] D. Marsh, Polarity and permeation profiles in lipid membranes, Proc. Natl. Acad. Sci. USA 98 (2001) 7777-7782.

[10] D. Kurad, G. Jeschke, D. Marsh, Lipid membrane polarity profiles by high-field EPR, Biophys. J. 85 (2003) 1025-1033.
[11] M.J. Swamy, M. Ramakrishnan, B. Angerstein, D. Marsh, Spin-label electron spin resonance studies on the mode of anchoring and vertical location of the $N$-acyl chain in $N$-acylphosphatidylethanolamines, Biochemistry 39 (2000) 12476-12484.

[12] K. Schorn, D. Marsh, Lipid chain dynamics and molecular location of diacylglycerol in hydrated binary mixtures with phosphatidylcholine: spin label ESR studies, Biochemistry 35 (1996) 3831-3836.

[13] Y.V.S. Rama Krishna, D. Marsh, Spin label ESR and ${ }^{31}$ P-NMR studies of the cubic inverted hexagonal phases of dimyristoylphosphatidylcholine/myristic acid $(1: 2 \mathrm{~mol} / \mathrm{mol})$ mixtures, Biochim. Biophys. Acta 1024 (1990) 89-94.

[14] R. Bartucci, A. Gambacorta, A. Gliozzi, D. Marsh, L. Sportelli, Bipolar tetraether lipids: chain flexibility and membrane polarity gradients from spin-label electron spin resonance, Biochemistry 44 (2005) 15017-15023.

[15] P. Hoffmann, K. Sandhoff, D. Marsh, Comparative dynamics and location of spin-labelled sphingomyelin and phosphatidylcholine in dimyristoyl phosphatidylcholine membranes studied by EPR spectroscopy, Biochim. Biophys. Acta 1468 (2000) 359-366.

[16] V. Monaco, F. Formaggio, M. Crisma, C. Toniolo, P. Hanson, G.L. Millhauser, Orientation and immersion depth of a helical lipopeptaibol in membranes using TOAC as an ESR probe, Biopolymers 50 (1999) 239-253.

[17] D. Marsh, M. Jost, C. Peggion, C. Toniolo, TOAC spin labels in the backbone of alamethicin: EPR studies in lipid membranes, Biophys. J. 92 (2007) 473-481.

[18] D. Marsh, M. Jost, C. Peggion, C. Toniolo, Lipid chainlength dependence for incorporation of alamethicin in membranes: EPR studies on TOAC-spin labelled analogues, Biophys. J. 92 (2007) 40024011.

[19] W.L. Hubbell, C. Altenbach, Site-directed spin-labeling of membrane proteins, in: S.H. White (Ed.), Membrane Protein Structure: Experimental Approaches, Oxford University Press, New York, 1994, pp. 224-248.

[20] W.L. Hubbell, A. Gross, R. Langen, M.A. Lietzow, Recent advances in site-directed spin labeling of proteins, Curr. Opin. Struct. Biol. 8 (1998) 649-656.

[21] W.L. Hubbell, D.S. Cafiso, C. Altenbach, Identifying conformational changes with site-directed spin labeling, Nat. Struct. Biol. 7 (2000) 735-739.

[22] H.J. Steinhoff, M. Pfeiffer, T. Rink, O. Burlon, M. Kurz, J. Riesle, E. Heuberger, K. Gerwert, D. Oesterhelt, Azide reduces the hydrophobic barrier of the bacteriorhodopsin proton channel, Biophys. J. 76 (1999) 2702-2710.

[23] C. Wegener, A. Savitsky, M. Pfeiffer, K. Möbius, H.J. Steinhoff, High-field EPR-detected shifts of magnetic tensor components of spin label side chains reveal protein conformational changes: the proton entrance channel of bacteriorhodopsin, Appl. Magn. Reson. 21 (2001) $441-452$.

[24] A. Savitsky, M. Kühn, D. Duche, K. Möbius, H.J. Steinhoff, Spontaneous refolding of the pore-forming colicin A toxin upon membrane association as studied by X-band and W-band high-field electron paramagnetic resonance spectroscopy, J. Phys. Chem. B 108 (2004) 9541-9548.

[25] R. Owenius, M. Engström, M. Lindgren, M. Huber, Influence of solvent polarity and hydrogen bonding on the EPR parameters of a nitroxide spin label studied by $9-\mathrm{GHz}$ and $95-\mathrm{GHz}$ EPR spectroscopy and DFT calculations, J. Phys. Chem. A 105 (2001) 1096710977.

[26] M. Karplus, G.K. Fraenkel, Theoretical interpretation of carbon-13 hyperfine interactions in electron spin resonance spectra, J. Chem. Phys. 35 (1961) 1312-1323.

[27] A.F. Gullá, D.E. Budil, Orientation dependence of electric field effects on the $g$ factor of nitroxides measured by $220 \mathrm{GHz}$ EPR, J. Phys. Chem. B 105 (2001) 8056-8063.

[28] R.N. Schwartz, M. Peric, S.A. Smith, B.L. Bales, Simple test of the effect of an electric field on the ${ }^{14} \mathrm{~N}$-hyperfine coupling constant in nitroxide spin probes, J. Phys. Chem. B 101 (1997) 8735-8739. 
[29] L. Onsager, Electric moments of molecules in liquids, J. Am. Chem. Soc. 58 (1936) 1486-1493.

[30] D. Marsh, Reaction fields and solvent dependence of the EPR parameters of nitroxides: the microenvironment of spin labels, J. Magn. Reson. 190 (2008) 60-67.

[31] A.H. Reddoch, S. Konishi, The solvent effect on di-tert-butyl nitroxide. A dipole-dipole model for polar solutes in polar solvents, J. Chem. Phys. 70 (1979) 2121-2130.

[32] H. Block, S.M. Walker, Modification of Onsager theory for a dielectric, Chem. Phys. Lett. 19 (1973) 363-364.

[33] I. Al-Bala'a, R.D. Bates Jr., Medium effects on ESR spectra in studies of hydrogen-bonded transient solvent-solute complexes, J. Magn. Reson. 73 (1987) 78-89.

[34] D. Marsh, Membrane water-penetration profiles from spin labels, Eur. Biophys. J. 31 (2002) 559-562.

[35] D. Marsh, Polarity contributions to hyperfine splittings of hydrogenbonded nitroxides - the microenvironment of spin labels, J. Magn. Reson. 157 (2002) 114-118.

[36] D.H. Whiffen, Information derived from anisotropic hyperfine couplings, J. Chim. Phys. 61 (1964) 1589-1591.

[37] A.H. Cohen, B.M. Hoffman, Hyperfine interactions in perturbed nitroxides, J. Am. Chem. Soc. 95 (1973) 2061-2062.

[38] M.A. Ondar, O.Ya. Grinberg, A.A. Dubinskii, Ya.S. Lebedev, Study of the effect of the medium on the magnetic-resonance parameters of nitroxyl radicals by high-resolution EPR spectroscopy, Sov. J. Chem. Phys. 3 (1985) 781-792.

[39] K. Möbius, A. Savitsky, A. Schnegg, M. Plato, M. Fuchs, High-field EPR spectroscopy applied to biological systems: characterization of molecular switches for electron and ion transfer, Phys. Chem. Chem. Phys. 7 (2005) 19-42.

[40] E.G. Rosantzev, Free Nitroxyl Radicals, Plenum, New YorkLondon, 1970

[41] A. Bondi, van der Waals volumes and radii, J. Phys. Chem. 68 (1964) $441-451$.
[42] P. Mukerjee, C. Ramachandran, R.A. Pyter, Solvent effects on the visible spectra of nitroxides and relation to nitrogen hyperfine splitting constants. Nonempirical polarity scales for aprotic hydroxylic solvents, J. Phys. Chem. 86 (1982) 3189-3197.

[43] R. Bartucci, D.A. Erilov, R. Guzzi, L. Sportelli, S.A. Dzuba, D. Marsh, Time-resolved electron spin resonance studies of spinlabelled lipids in membranes, Chem. Phys. Lipids 141 (2006) 142157.

[44] D. Marsh, D. Kurad, V.A. Livshits, High-field spin label EPR of lipid membranes, Magn. Reson. Chem. 43 (2005) S20-S25.

[45] R. Bartucci, R. Guzzi, D. Marsh, L. Sportelli, Intramembrane polarity by electron spin echo spectroscopy of labeled lipids, Biophys. J. 84 (2003) 1025-1030.

[46] D.A. Erilov, R. Bartucci, R. Guzzi, A.A. Shubin, A.G. Maryasov, D. Marsh, S.A. Dzuba, L. Sportelli, Water concentration profiles in membranes measured by ESEEM of spin-labeled lipids, J. Phys. Chem. B 109 (2005) 12003-12013.

[47] J.F. Nagle, S. Tristram-Nagle, Structure of lipid bilayers, Biochim. Biophys. Acta 1469 (2000) 159-195.

[48] C.J.F. Böttcher, Theory of Electric Polarization, Elsevier Scientific Publishing Company, Amsterdam, 1973.

[49] K.A. Earle, J.K. Moscicki, M.T. Ge, D.E. Budil, J.H. Freed, 250$\mathrm{GHz}$ electron spin resonance studies of polarity gradients along the aliphatic chains in phospholipid membranes, Biophys. J. 66 (1994) 1213-1221.

[50] L.A. Carpino, 1-Hydroxy-7-azabenzotriazole. An efficient peptide coupling additive, J. Am. Chem. Soc. 115 (1993) 4397-4398.

[51] J.F.W. Keana, S.B. Keana, D. Beetham, A new versatile ketone spin label, J. Am. Chem. Soc. 89 (1967) 3055-3056.

[52] B.G. Segal, M. Kaplan, G.K. Fraenkel, Measurement of $g$-values in electron spin resonance spectra of free radicals, J. Chem. Phys. 43 (1965) 4191-4200.

[53] R.D. Allendoerfer, Comment on determination of absolute $g$-values, J. Chem. Phys. 55 (1971) 3615. 